\title{
PEPC sensitization with polymethine dyes: 1. Squarylium dyes of indole row as efficient sensitizers for red region of optical spectrum
}

\author{
M.Yu. Bazhenov', P.I. Golod ${ }^{\mathrm{b}}$, V.V. Grabovskyya ${ }^{\mathrm{a}}$, V.V. Kurdyukov ${ }^{\mathrm{c}}$, A.I. Tolmachev \\ A.Ya. Il'chenko , N.I. Sokolov ${ }^{\mathrm{b}}$, G.Zahaykevich ${ }^{\mathrm{d}}$ \\ ${ }^{a} T A V-A M$, Office 4, 11 Stritens'ka str., Kyiv, Ukraine. Phone +380 (44) 212-5796, \\ tavam@ukrpack.net \\ ${ }^{b}$ Laboratory of Nonlinear Optics, Department of Natural Science, National University "Kyiv Mohyla Academy", 2 Skovoroda str., \\ Kyiv, 254114, Ukraine. \\ Phone +380 (44) 416-6068 \\ 'Institute of Organic Chemistry NASU, Kyiv, Ukraine. \\ iohkiev@ukrpack.net \\ ${ }^{d}$ Tavex America Inc., 14 Garden Rd., Natick, MA 01760. Phone (508) 620-7555, \\ gz@tavexamerica.com
}

\begin{abstract}
Phase hologram recording is based on photoconductivity which appears in polymer molecular semiconductor film being exposed to light [1]. Photocurrent threshold depends on energy gap between valence and conduction bands of a photoconductor (PC). Energy gap of PCs based on poly-N-epoxypropylcarbazole (PEPC) which are widely used for hologram recording is rather large. That is why recording on pure PEPC film may be only in UV range of optical spectra. Thus, sensitization of PEPC with different dyes to make it sensitive to visible range is usually used for practice. Nowadays structural PEPC sensitization with fluorene electron acceptors is widely used for holographic recording material synthesis [2]. There are few publications about organic dye sensitization of PEPC due to low efficiency of first dyes used. Main obstacle to practical use of dyes like rhodamine $6 \mathrm{G}$, malachite green and other 3-phenilmethans, is low diffraction efficiency $(\eta)$ of hologram due to large charges thermo-relaxation in single-layer PEPC films.

We have investigated some polymethine dyes like squarylium with indole residues for PEPC sensitization in red range of visible spectrum. These dyes are under intense interest due to its promising usefulness for xerography and optical recording. Particularity of such dyes is its intra-ionoide, actually bipolar, structure.
\end{abstract}

Keywords: recording material, structural sensitization, single-layer film.

Paper received 14.04.00; revised manuscript received 14.05.00; accepted for publication 16.06.00.

\section{Introduction}

It is well-known that photosensitization of PC with organic dyes will be successful if upper occupied level of dye is lower than valence band of PC. Being exposed to light dye molecule may capture electron from $\mathrm{PC}$ and make it hole conductive [1]. That is why some polymethine dyes with pyril and thiopyril residues (that caused lower top occupied electron level than of pure polymethine) which are characterized like rather bad electron donors [3] were used for PEPC sensitization [4]. Polymethine dyes including pyril derivatives in contrast to rhodamine $6 \mathrm{G}$ and triphenyl are usually plain. It may enable these molecules to get closer to PC molecules and thus to get more expressed sensitization effect. But such dyes have salt-like structure and are not well dissolved in polar solvents widely used for making PC films. All mentioned above make difficult such polymethine dyes for practical usage.

In this paper first results of PEPC sensitization in red range of optical spectra with squarylium dyes are presented. These dyes are under intense interest due to its promising usefulness for xerography and optical recording [5]. They have a strong wide absorption band. Their advantage is nonsalt-like structure, so they can be dis- 


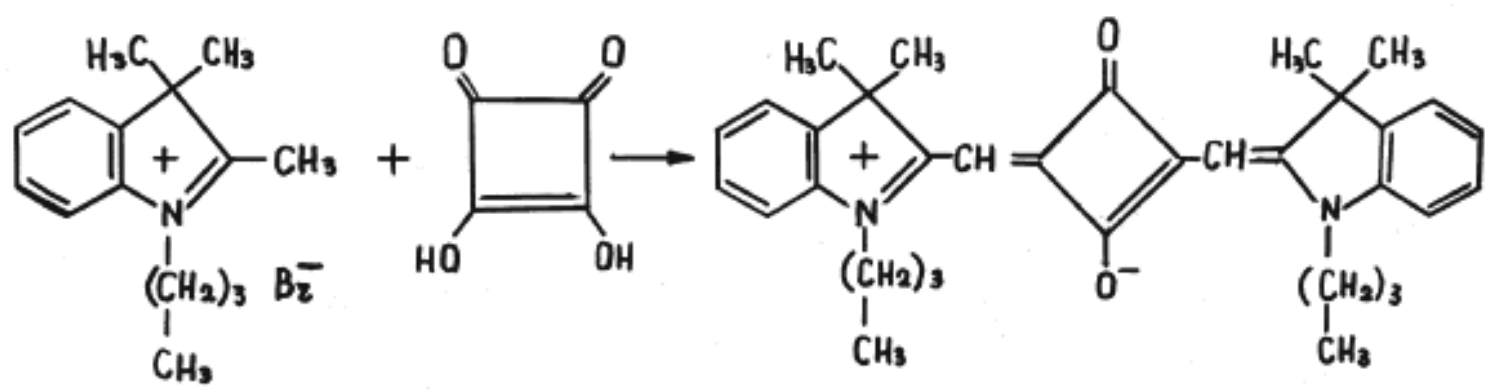

Fig. 1. The synthesis scheme of the dye \#73 - 1(N-butyl)-2,3,3-trimethylindole bromide with square acid.

solved in conventional solvents. It was determined earlier using X-ray analysis, IR and paramagnetic resonance spectroscopy that dye molecules are almost flat, have a centro-symmetric (close to bipolar) structure [6]. We have investigated derivatives of 2,3,3-trimethylindole, particularly the dye \#73 as a result of condensation of $1(\mathrm{~N}$ butyl)-2,3,3-trimethylindole bromide with square acid (see Fig.1).

$\mathrm{N}$-butyl groups were added to nitrogen atoms of heterocyclic dye residues for increasing its solubility in lowpolar toluene, which was used for single-layer thin film formation.

\section{Experiment}

We have estimated energies of top occupied level of PEPC and the dye \#73 as well as their electron structure and absorption spectra using the quantum-chemical PPP method [7]. We have used modified set of parameters, namely: ionization potentials, where 19,21 and $11.16 \mathrm{eV}$ for nitrogen, oxygen and carbon atoms, respectively. Onecentered integrals $\gamma_{r r} 7.5,6.5$ and $6.5 \mathrm{eV}$ and two-centered integrals $\gamma_{r s}$ were calculated by the Mataga-Nishimoto method. We modified parameters for getting closer with calculation to measured absorption bands for cyanin dyes. So, absorption maximums for indole mono- and dicarbocyanin dyes are at 556 and $630 \mathrm{~nm}$ (calculation) and $548 \mathrm{~nm}$ (exp. [8]) and $638 \mathrm{~nm}$ (exp. [9, 10]), respectively. For indole squarylium dye absorption maximum is at $635 \mathrm{~nm}$ (calc.) and $629 \mathrm{~nm}$ (exp. [9]), $630 \mathrm{~nm}$ (exp. [10]) and $633 \mathrm{~nm}$ (exp. [11]).

It was found that upper occupied electron level of $\mathrm{PEPC}$ is at $-8.8 \mathrm{eV}$ and $-10.3 \mathrm{eV}$ for the indole squarylim dye \#73. Thus, the dye \#73 may be used as efficient sensitizer of PEPC, especially in a red region of the optical spectrum.

Electron absorption spectra of toluene solutions of PEPC (curve 1), $\beta$-naphthyl-glycide ether ( $\beta$-NGE) with glycidecarbozole (curve 2 ) and low-molecular $\beta$-NGE [12] (curve 3 ) all sensitized with the dye \#73 are shown in Fig. 2.

Low-molecular $\beta$-NGEs are synthesized in the Laboratory of monomer and polymer chemistry of Taras
Shevchenko National University. They differ from PEPC generally by size of integrated conjugated $\pi$-electrons of its chromophor groups. Absorption spectra were recorded on spectrophotometer SF-26. Concentrations of all polymers in toluene were 2.3 mass \% with the dye \#73 concentration 0.2 mass $\%$. One can see that all spectra have the same shape so absorption is caused by dye. The size of integrated conjugated $\pi$-electrons of its chromophor groups does not affect on absorption as it is in the case of structural sensitization of PC with fluorine dyes [13]. Thereby the dye \#73 does not chemically interact with these PCs.

Addition of the dye \#73 into single-layer composition of organic PC allows to get comparative high hologram diffractive efficiency on PEPC-based films with softening temperature below $85^{\circ} \mathrm{C}$ and almost have no influence on thermorelaxation of surface charge.

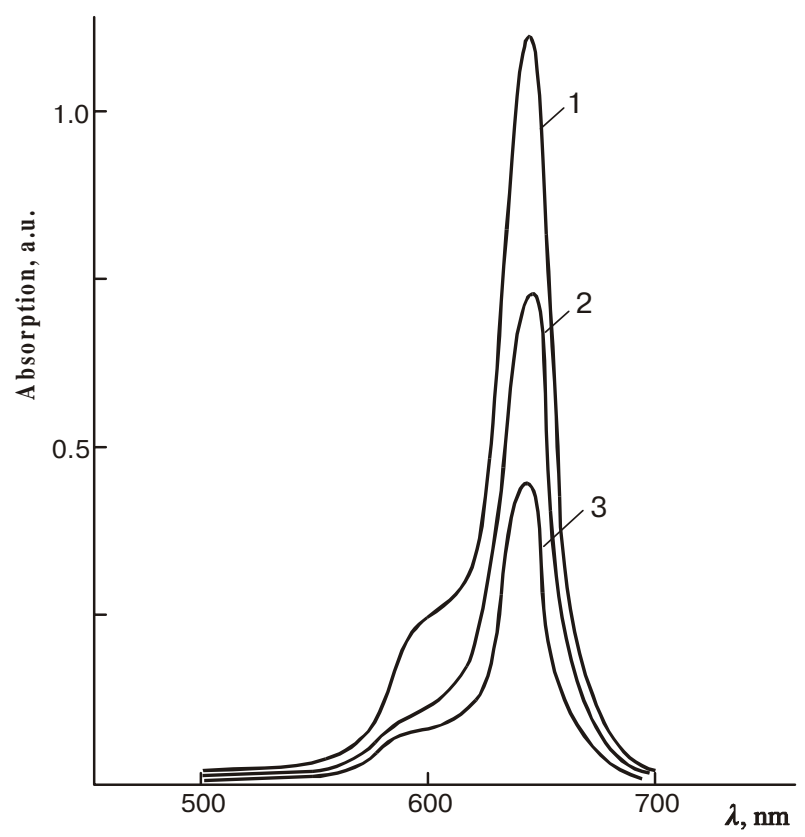

Fig. 2. Absorption spectra of the dye \#73 in toluene (curve 1), concentration $C=4.72 \mathrm{~mol} \times \mathrm{dm}^{-3}, \lambda_{\max }=644 \mathrm{~nm}$. 


\section{M.Yu. Bazhenov et al.: PEPC sensitization with polymethine dyes}

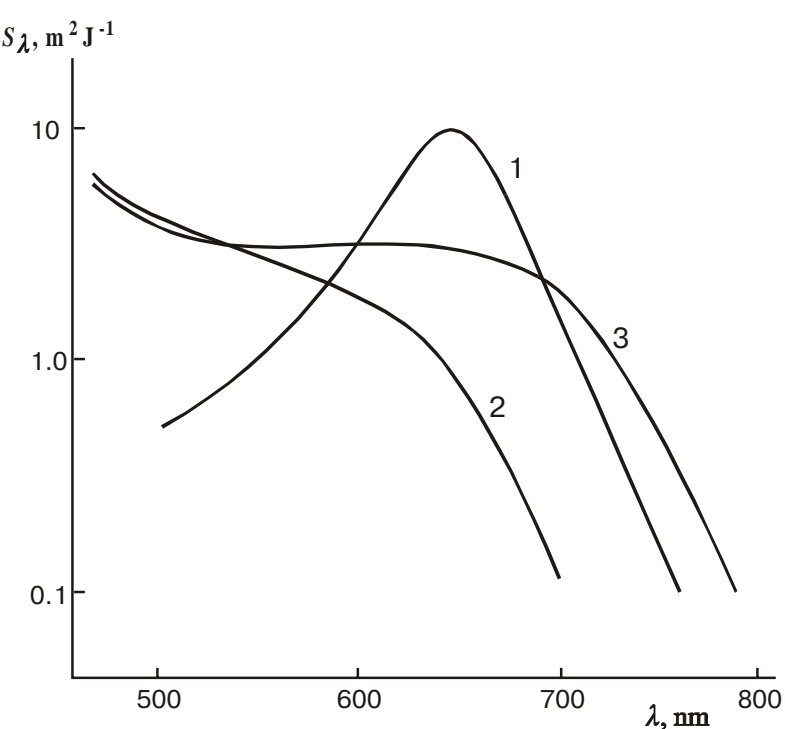

Fig. 3. Spectral distribution of electrophotographic sensitivity $\mathrm{S}_{\lambda}$ of different PEPC films.

Spectral dependencies of electrophotographic sensitivity $S_{\lambda}$, determined as $20 \%$ decay of surface potential compared with the initial one $\left(S_{\triangle V / V}=0.2\right)$ under light in electric field $E=1.2 \times 10^{8} \mathrm{~V} / \mathrm{m}$ for PEPC +0.2 mass $\%$ dye \#73 (curve 1), PEPC +5 mass $\%$ trinitrofluorene (curve 2) and PEPC +3 mass $\%$ uidicil ether 9-dicianmethylene2,7-dinitrofluorene-4-carbazole acid (curve 3) are shown in Fig. 3.

Last two curves are shown for comparison. At $633 \mathrm{~nm}$ $\mathrm{S}_{\triangle \mathrm{V} / \mathrm{V}}$ for PEPC +0.2 mass $\%$ dye $\# 73$ is higher than that for all known PEPC+fluorene dyes compositions.

\section{Conclusion}

Real holographic sensitivity $S_{\eta}$ of PEPC +0.2 mass \% dye \#73 is higher than that for convention compositions and is $S_{\eta=1 \%} \geq 150 \mathrm{~m}^{2} / \mathrm{J}$ for single-layer film with He-Ne laser hologram making. Thermorelaxation of a surface potential with the optimal dye concentration $(0.2-0.4 \mathrm{~mol} . \%)$ is low and allows to get maximum diffractive efficiency of phase-relief holograms.

Measurements of real holographic sensitivity and hologram registration were carried out with TCC-2 holographic camera [14]. Single-layer polymer semiconductor compositions have well investigated features, a high hologram registration dynamic range and can be used for diffusive object registration with object-to-reference beam ratio up to 2000 (reference beam power density was about $30 \mu \mathrm{W} / \mathrm{cm}^{2}$ ). So, the object beam power density may be as low as $0.015 \mu \mathrm{W} / \mathrm{cm}^{2}$.

Sensitization of PC like PEPC with polymethine dyes of the indole row may be more useful in comparison with sensitization by electron acceptors in some cases. Especially for making PCs sensitive in a red range of the optical spectrum, where a powerful low-cost diode laser may be used for phase hologram recording.

\section{References}

1. N.G. Kuvshinsky, N.A. Davidenko, V.M. Komko. Physics of amorphous molecular semiconductors, Lybid', Kyiv, 1994.

2. I.F. Perepichka, D.D. Mysyk and N.I. Sokolov, Photoconductivity of carbazole-containing polymers sensitized by electron acceptors. In: Current Trends in Polymer Photochemistry, ed. N.S. Allen, M. Edge, J.R. Bellobono and E. Selli, Ellis Horwood, London, 1995, ch. 18, p. 318.

3. M.A. Kudinova, N.A. Derevyanko, G.G. Dyadyusha, A.A. Ishchenko, A.I. Tolmachev, Pyrilcyanin. 12. Nonsymmetric pyril-2-cyanin. In: Chemistry of hetero-cycling compounds, 1980,7, p. 903.

4. M.S. Borodkina, I.A. Malakhova, T.V. Chel'cova, Poly-Nvinylcarbazole sensitization. In: Photonics of organic semiconductors, Naukova dumka, Kyiv, 1977, p. 114.

5. S.H. Kim, S.H. Hwang, Synthesis and photostability of functional squarylium dyes // Dyes a pigments, 35, p. 111 (1997).

6. A.I. Tolmachev, V.V. Kurdyukov, M.A. Kudinova, E.B. Rusanov, A.N. Chernega, Molecular and crystal structure of squarylium dyes, derivatives of pyril // Journal of structural chemistry, to be published.

7. R. Zagranik, P. Polak. Basics of quantum chemistry, Mir, Moscow, 1979.

8. F.M. Hamer, A general method for preparation of carbocyanin dyes // J. Chem. Soc., p. 2796-2804 (1927).

9. S. Yasen, M. Matsuoka, T. Kitao, Synthesis and some properties of infrared-absorbing croconium and related dyes // Dyes a pigments, 10, p. 13 (1988).

10. H.-E.Sprenger, W. Ziegenbein, Das cyclobuten-diyliumkation ein neuartiger chromophor aus quadratsaure // Angew. Chem., 79, p. 581 (1967).

11. S.H. Kim, S.K. Han, J.J. Kim, S.H. Hwang, Synthesis and characteristics of new dithiosquarylium dyes // Dyes a pigments, 39, p.77, (1998).

12. Yu.P. Getmanchuk, V.A. Kudrenko, N.I. Sokolov, Synthesis and useful possibility of naphthalene-containing polymers as photothermoplastic registration media // Ukrainian Chemical Journal, 56 (12), (1990).

13. Yu.P. Getmanchuk, N.I. Sokolov, About structure and spectral sensitivity of some polymer photoconductors, sensitized with different electron acceptor. In: Fundamentals of optical memory and media, Kiev, "Vysshaya skhola", Issue 14, p. 1119, 1983.

14. M.Yu. Bazhenov, V.V. Grabovski, A.V. Stolyarenko, Realtime holographic camera TCC-2 // Proc. SPIE, 3055, p. 255259, 1996. 